Проблеми математичного моделювання та теорії диференціальних рівнянь

УДК 517.9

\title{
ЗАДАЧІ АПРІОРНОГО СИНТЕЗУ ОПТИМАЛЬНИХ КЕРУВАНЬ ДЛЯ ВИРОДЖЕНИХ ПАРАБОЛІЧНИХ РІВНЯНЬ
}

\section{І. Г. Баланенко, П. І. Когут}

Дніпропетровсъжий національний університет ім. Олеся Гончара, кафедра диференціальних рівнянь, вул. Казакова, 18/14, ДНУ, 49010, Дніпропетровсък, E-mail: balanenko-ig@rambler.ru,p.kogut@i.ua

Досліджено один клас задач оптимального керування для виродженого параболічного рівняння з крайовими умовами Діріхле на межі області та з апріорі заданою структурою оберненого зв'язку. Показано, що для таких задач існують оптимальні розв'язки у вагових просторах Соболєва за умови компактності операторів, які формують обернений зв'язок.

Ключові слова: оптимальне керування, нерівність Харді - Пуанкаре, параболічне рівняння, апріорний синтез.

\section{1. Вступ}

Основним об'єктом досліджень виступає задача оптимального керування виродженим параболічним рівнянням

$$
\rho(x) \dot{y}-\nu \operatorname{div}(\rho(x) \nabla y)=f(t, x)+p(t, x)
$$

з крайовими умовами Діріхле на межі області за умови, що є апріорі задана структура керування у формі оберненого зв'язку

$$
p(t, x)=\sum_{j=1}^{M} u_{j}(t) \mathcal{M}_{j}(y),
$$

де $\mathcal{M}_{j}: W_{0}^{1,2}(\Omega, \rho d x) \rightarrow\left(W_{0}^{1,2}(\Omega, \rho d x)\right)^{*}$ - лінійні неперервні оператори, а функції $u=\left[u_{1}, \ldots, u_{M}\right]^{T} \in L^{2}(0, T)^{M}$ підлягають визначенню.

Характерною рисою вироджених параболічних рівнянь та пов'язаних із ними початково-крайових задач є та обставина, що проблема їх розв' язності суттєво залежить від властивостей вагової функції $\rho$ (див., напр., [3,7]). Той факт, що функція $\rho$ може бути необмеженою на області $\Omega$ чи досягати нуля на підмножинах нульової міри Лебега, означає, що диференціальний оператор $\operatorname{div}(\rho(x) \nabla)$ втрачає властивість коерцитивності та неперервності на

(C) I. Г. Баланенко, П. I. Когут, 2014 
$L^{2}\left(0, T ; H_{0}^{1}(\Omega)\right)$. Як наслідок, наведені задачі можуть успадковувати неєдиність слабких розв'язків та інші ефекти, притаманні некоректним задачам математичної фізики.

Як відомо (див., напр., [1,2]), базовим у теорії початково-крайових задач для вироджених параболічних рівнянь $є$ поняття вагового простору Соболєва $W_{0}^{1,2}(\Omega ; \rho d x)$. Оскільки простір фінітних функцій $C_{0}^{\infty}(\Omega)$ не є в загальному випадку щільний у $W_{0}^{1,2}(\Omega ; \rho d x)$, то ця обставина породжує суттєві труднощі в обгрунтуванні проблеми єдиності слабких розв'язків таких задач та отриманні відповідних апріорних оцінок для них. У зв'язку з цим автори показують, що таке обгрунтування стає можливим, якщо вагову функцію $\rho=\rho(x)$ наділити певними додатковими властивостями, що не виводять її 3 класу необмежених та вироджених на $\Omega$ функцій. Такою умовою $є$ приналежність $\rho$ до класу функцій потенціального типу. В результаті, залучивши нерівність типу Харді - Пуанкаре та умови компактності для операторів $\mathcal{M}_{j}$, стало можливим установити, що задача апріорного синтезу оптимального керування для вихідного виродженого параболічного рівняння має єдиний оптимальний розв'язок у вагових просторах Соболєва. Для цього розв'язку отримано та обгрунтовано апріорні оцінки.

Окрім цього, у роботі розглянуто геометричне узагальнення задачі оптимального керування з апріорі заданою структурою оберненого зв'язку, яке полягає в тому, що оператори $\mathcal{M}_{j}$ обираються у вигляді

$$
\mathcal{M}_{j}(\phi)=c \sqrt{\rho(x)} \int_{B\left(b_{j}, r\right)} \phi(s) \sqrt{\rho}(s) d s, \quad \forall \phi \in W_{0}^{1,2}(\Omega, \rho d x),
$$

де центри $b_{j}$ "позиціювання" куль $B\left(b_{j}, r\right)$ вважають невідомими і вони підлягають визначенню. Показано, що така задача має розв'язок за умови, що примежовий шар множини $\Omega \in$ недопустимий для вибору точок $b_{j}$.

\section{2. Основні позначення та факти}

Нехай $\Omega \subset \mathbb{R}^{N}(N \geqslant 3)$ - обмежена відкрита підмножина з достатньо регулярною межею $\partial \Omega$. Нехай $Q=(0, T) \times \Omega \in$ циліндром в $\mathbb{R}^{1} \times \mathbb{R}^{N}$, де $T<+\infty$. Через $\Sigma=(0, T) \times \partial \Omega$ позначимо його бокову поверхню. Нехай $H_{0}^{1}(\Omega)$ є простором Соболєва, який утворено замиканням множини $C_{0}^{\infty}(\Omega)$ за нормою

$$
\|y\|_{H_{0}^{1}(\Omega)}=\left(\int_{\Omega}|\nabla y(x)|_{\mathbb{R}^{N}}^{2} d x\right)^{1 / 2}
$$

Нехай є заданою функція $\rho: \Omega \rightarrow \mathbb{R}$ така, що: $\rho(x)>0$ майже скрізь (м.с.) на $\Omega$,

$$
\rho \in L^{1}(\Omega), \rho^{-1} \in L^{1}(\Omega), \nabla|\ln \rho| \in L^{2}\left(\Omega ; \mathbb{R}^{N}\right) \text { i } \rho+\rho^{-1} \notin L^{\infty}(\Omega) .
$$


Надалі будемо пов' язувати з функцією $\rho$ такі вагові простори $L^{2}(\Omega, \rho d x)$, $L^{2}\left(\Omega, \rho^{-1} d x\right)$ та $W_{0}^{1,2}(\Omega, \rho d x)$, де через $W_{0}^{1,2}(\Omega, \rho d x)$ позначено ваговий простір Соболєва, утворений елементами з $W_{0}^{1,1}(\Omega)$, для яких є скінченна норма

$$
\|y\|_{W_{0}^{1,2}(\Omega, \rho d x)}:=\left(\int_{\Omega} y^{2} \rho d x+\int_{\Omega}|\nabla y|_{\mathbb{R}^{N}}^{2} \rho d x\right)^{1 / 2} .
$$

Означення 2.1. Будемо казати, що $\rho: \Omega \rightarrow \mathbb{R}_{+} \in$ ваговою функцією потенціального типу, якщо

(i) $\rho>0$ майже скрізь (м.с.) на $\Omega, \rho \in L^{1}(\Omega), \rho^{-1} \in L^{1}(\Omega)$;

(ii) існує підобласть $\Omega_{*} \subset \Omega$ така, що dist $\left(\partial \Omega, \partial \Omega_{*}\right) \geqslant \delta>0$,

$$
\rho \in C^{1}\left(\overline{\Omega \backslash \Omega_{*}}\right) \text { і } \rho(x) \geqslant \sigma \quad \text { на } \Omega \backslash \Omega_{*} \quad \text { за деякого } \sigma>0 \text {; }
$$

(iii) існують сталі $C>0, \lambda<\lambda_{*}:=(N-2)^{2} / 4$ та система внутрішніх точок $\left\{x_{1}^{*}, x_{2}^{*}, \ldots, x_{K}^{*}\right\}$ множини $\left.\Omega\right)$ такі, що

$$
-C \leqslant V(x) \leqslant \frac{2 \lambda}{K}\left(\sum_{i=1}^{K} \frac{1}{\left|x-x_{i}^{*}\right|_{\mathbb{R}^{N}}^{2}}\right), \quad \forall x \in \Omega,
$$

де позначено $V(x)=-\Delta \ln \rho(x)-\frac{1}{2}|\nabla \ln \rho(x)|_{\mathbb{R}^{N}}^{2}$.

\section{3. Постановка задачі оптимального керування}

Нехай $y_{a d} \in L^{2}(Q), f \in L^{2}\left(0, T ; L^{2}\left(\Omega, \rho^{-1} d x\right)\right)$, та $y_{0} \in L^{2}(\Omega)-$ задані функції. Нехай $M \in \mathbb{N}$ та $\nu \neq 0$ - фіксовані сталі. Нехай $U_{\partial}-$ непорожня опукла замкнена підмножина в $L^{2}(0, T)^{M}$.

Розглянемо в циліндрі $Q=(0, T) \times \Omega$ наступну задачу оптимального керування для виродженого параболічного рівняння з апріорі заданою структурою закону оберненого зв'язку:

$$
\begin{gathered}
I(u, y)=\int_{0}^{T}\left\|y(t, \cdot)-\frac{y_{a d}(t, \cdot)}{\sqrt{\rho}}\right\|_{L^{2}(\Omega, \rho d x)}^{2} d t+\sum_{j=1}^{M} N_{j} \int_{0}^{T}\left|u_{j}(t)\right|^{2} d t \longrightarrow \inf \\
\rho(x) \dot{y}-\nu \operatorname{div}(\rho(x) \nabla y)=f(t, x)+p(t, x) \quad \text { в } Q=(0, T) \times \Omega \\
y(t, x)=0 \quad \text { на }(0, T) \times \partial \Omega \\
\sqrt{\rho(x)} y(0, x)=y_{0} \quad \text { майже скрізь на } \Omega \\
p(t, x)=\sum_{j=1}^{M} u_{j}(t) \mathcal{M}_{j}(y), \quad u=\left[u_{1}, \ldots, u_{M}\right]^{T} \in U_{\partial}
\end{gathered}
$$


Тут $N_{j}>0$ - задані сталі, $\mathcal{M}_{j}: W_{0}^{1,2}(\Omega, \rho d x) \rightarrow\left(W_{0}^{1,2}(\Omega, \rho d x)\right)^{*}-$ лінійні неперервні оператори такі, що

$$
\left|\left\langle\mathcal{M}_{j}(y), y\right\rangle_{\left(W_{0}^{1,2}(\Omega, \rho d x)\right)^{*} ; W_{0}^{1,2}(\Omega, \rho d x)}\right| \leqslant C\|y\|_{W_{0}^{1,2}(\Omega, \rho d x)}\|y\|_{L^{2}(\Omega, \rho d x)}
$$

для всіх $j=1, \ldots, M$ зі сталою $C>0$, яка не залежить від $y$ та $j$.

Для початку розглянемо задачу оптимального керування (3.1)-(3.5), яка полягає у визначенні функцій $\left(u_{1}^{0}, \ldots, u_{M}^{0}, y^{0}\right) \in L^{2}(0, T)^{M} \times L^{2}\left(0, T ; \mathcal{W}_{\rho}\right)$ (надалі їх будемо називати оптимальними), які в слабкому сенсі задовольняють співвідношення (3.2)-(3.5) і на яких функціонал (3.1) досягає свого найменшого можливого значення.

Пов'яжемо 3 початково-крайовою задачею (3.2)-(3.4) лінійний оператор $A: W_{0}^{1,2}(\Omega, \rho d x) \rightarrow\left(W_{0}^{1,2}(\Omega, \rho d x)\right)^{*}$, залучивши правило:

$$
\langle A y, v\rangle_{\left(W_{0}^{1,2}(\Omega, \rho d x)\right)^{*} ; W_{0}^{1,2}(\Omega, \rho d x)}=\int_{\Omega}(\nabla y, \nabla v)_{\mathbb{R}^{N}} \rho d x \quad \forall v \in W_{0}^{1,2}(\Omega, \rho d x) .
$$

Ясно, що $A y=-\operatorname{div}(\rho(x) \nabla y)$, а отже, з огляду вихідних припущень оператор $A: W_{0}^{1,2}(\Omega, \rho d x) \rightarrow\left(W_{0}^{1,2}(\Omega, \rho d x)\right)^{*}$ не задовольняє умову коерцитивності. Дійсно, оскільки

$$
\langle A(y), y\rangle_{\left(W_{0}^{1,2}(\Omega, \rho d x)\right)^{*} ; W_{0}^{1,2}(\Omega, \rho d x)}=\int_{\Omega}|\nabla y|_{\mathbb{R}^{N}}^{2} \rho d x \neq\|y\|_{W_{0}^{1,2}(\Omega, \rho d x)}^{2},
$$

то немає жодних підстав стверджувати виконання наступної умови:

$$
\frac{\left\langle A(y), y-v_{0}\right\rangle_{W_{0}^{1,2}(\Omega, \rho d x)}}{\|y\|_{W_{0}^{1,2}(\Omega, \rho d x)}} \rightarrow+\infty \text { при }\|y\|_{W_{0}^{1,2}(\Omega, \rho d x)} \rightarrow \infty .
$$

У результаті проблема існування та єдиності розв'язків початково-крайової задачі (3.1)-(3.5) на класі допустимих керувань залишається відкритим питанням.

Таким чином, характерною рисою задачі оптимального керування (3.1)(3.5) є те, що за певного вибору функції $\rho$ з властивостями (2.1) відповідна множина допустимих розв'язків задачі (3.1)-(3.5) може виявитися порожньою.

\section{4. Попередній аналіз задачі оптимального керування (3.1)-(3.5)}

Твердження 4.1. Для довільного елемента $y \in W_{0}^{1,2}(\Omega, \rho d x)$ має місце подання $y=\frac{z}{\sqrt{\rho}}$, де $z \in W_{0}^{1,1}(\Omega) \cap L^{2}(\Omega)$.

Доведення. Зафіксуємо довільний елемент $y \in W_{0}^{1,2}(\Omega, \rho d x)$ і покажемо, що $z=y \sqrt{\rho} \in W_{0}^{1,1}(\Omega) \cap L^{2}(\Omega)$. Дійсно, маємо

$$
\|z\|_{L^{2}(\Omega)}^{2}=\int_{\Omega}|y \sqrt{\rho}|^{2} d x=\left(\int_{\Omega} y^{2} \rho d x\right) \leqslant C\|y\|_{W_{0}^{1,2}(\Omega, \rho d x)}^{2} .
$$


Далі, залучаючи перетворення

$$
\nabla(\sqrt{\rho} y)=y \frac{1}{2 \sqrt{\rho}} \nabla \rho+\sqrt{\rho} \nabla y=\sqrt{\rho}\left(\nabla y+\frac{1}{2 \rho} y \nabla \rho\right)=\sqrt{\rho}\left(\nabla y+\frac{y}{2} \nabla \ln \rho\right)
$$

та властивості вагової функції $\rho(x)$, отримаємо

$$
\begin{aligned}
\|\nabla z\|_{L^{1}\left(\Omega ; \mathbb{R}^{N}\right)} & =\int_{\Omega}\left|\sqrt{\rho}\left(\nabla y+\frac{y}{2} \nabla \ln \rho\right)\right|_{\mathbb{R}^{N}} d x \\
& \leqslant \int_{\Omega}|\sqrt{\rho} \nabla y|_{\mathbb{R}^{N}} d x+\frac{1}{2} \int_{\Omega}|\sqrt{\rho} y \nabla \ln \rho|_{\mathbb{R}^{N}} d x \\
& \leqslant\left(\int_{\Omega} \rho|\nabla y|_{\mathbb{R}^{N}} d x\right)^{1 / 2}|\Omega|^{1 / 2}+\frac{1}{2}\left(\int_{\Omega} y^{2} \rho d x\right)^{1 / 2}\left(\int_{\Omega}|\nabla \ln \rho|_{\mathbb{R}^{N}}^{2}\right)^{1 / 2} \\
& \leqslant C_{1}\|y\|_{W_{0}^{1,2}(\Omega, \rho d x)} .
\end{aligned}
$$

Таким чином, $z \in W^{1,1}(\Omega) \cap L^{2}(\Omega)$. Окрім того, елемент $z=y \sqrt{\rho}$ успадковує властивості сліду вздовж межі області $\partial \Omega$ від елемента $y$, і нарешті, отримаємо $z \in W_{0}^{1,1}(\Omega) \cap L^{2}(\Omega)$. Твердження доведено.

Насправді, відображення $\varphi: W_{0}^{1,2}(\Omega, \rho d x) \rightarrow W_{0}^{1,1}(\Omega) \cap L^{2}(\Omega)$, що визначається як $\varphi(y)=y \sqrt{\rho}$, не є сюр'єктивне. Проте у просторі $W_{0}^{1,1}(\Omega) \cap L^{2}(\Omega)$ множина його образів $\varphi\left(W_{0}^{1,2}(\Omega, \rho d x)\right)$ є щільна. Легко бачити, що для довільного $z \in C_{0}^{\infty}(\Omega) \subset W_{0}^{1,1}(\Omega) \cap L^{2}(\Omega)$, маємо $\frac{z}{\sqrt{\rho}} \in W_{0}^{1,2}(\Omega, \rho d x)$. Дійсно,

$$
\begin{aligned}
& \left\|\frac{z}{\sqrt{\rho}}\right\|_{W_{0}^{1,2}(\Omega, \rho d x)}=\int_{\Omega} z^{2} \rho d x+\int_{\Omega}\left|\frac{1}{\sqrt{\rho}} \nabla z-\frac{z}{2 \sqrt{\rho}} \nabla \rho\right|_{\mathbb{R}^{N}}^{2} \rho d x \\
& \leqslant\left\|z^{2}\right\|_{C(\Omega)} \int_{\Omega} \rho d x+2 \int_{\Omega}|\nabla z|_{\mathbb{R}^{N}}^{2} d x+\frac{1}{2} \int_{\Omega} z^{2}|\ln \rho|_{\mathbb{R}^{N}}^{2} d x \\
& \leqslant\left\|z^{2}\right\|_{C(\Omega)}\|\rho\|_{L^{1}(\Omega)}+2\left\|\left.\nabla z\right|_{\mathbb{R}^{N}} ^{2}\right\|_{C(\Omega)}+\frac{1}{2}\left\|z^{2}\right\|_{C(\Omega)}\|\nabla \ln \rho\|_{L^{2}\left(\Omega ; \mathbb{R}^{N}\right)}^{2}<\infty
\end{aligned}
$$

Таким чином, як очевидний наслідок попереднього результату та неперервності вкладення $H_{0}^{1}(\Omega) \subset W_{0}^{1,1}(\Omega) \cap L^{2}(\Omega)$, отримаємо наступний результат.

Наслідок 4.1. Існуе щілъна множина $D_{\rho} \subset H_{0}^{1}(\Omega)$, така що

$$
\frac{z}{\sqrt{\rho}} \in W_{0}^{1,2}(\Omega, \rho d x), \forall z \in D_{\rho}
$$

Беручи до уваги дане твердження, введемо до розгляду таке лінійне відображення:

$$
\mathfrak{F}: D_{\rho} \subset H_{0}^{1}(\Omega) \rightarrow W_{0}^{1,2}(\Omega, \rho d x), \quad \text { де } \quad \mathfrak{F} z=\frac{z}{\sqrt{\rho}} .
$$


Оскільки область визначення $D_{\rho}$ даного відображення є щільною множиною банахового простору $H_{0}^{1}(\Omega)$, то для $\mathfrak{F}$, як щільно визначеного оператора, існує спряжений оператор $\mathfrak{F}^{*}: D\left(\mathfrak{F}^{*}\right) \subset W^{-1,2}\left(\Omega, \rho^{-1} d x\right) \rightarrow H^{-1}(\Omega)$ такий, що

$$
\left\langle\mathfrak{F}^{*} v, z\right\rangle_{H^{-1}(\Omega) ; H_{0}^{1}(\Omega)}=\langle v, \mathfrak{F} z\rangle_{\left(W_{0}^{1,2}(\Omega, \rho d x)\right)^{*} ; W_{0}^{1,2}(\Omega, \rho d x)}, \forall z \in D_{\rho} \mathrm{i} \forall v \in D\left(\mathfrak{F}^{*}\right),
$$

де

$$
D\left(\mathfrak{F}^{*}\right)=\left\{v \in W^{-1,2}\left(\Omega, \rho^{-1} d x\right) \mid \begin{array}{c}
\exists C>0 \text { таких, що для всіх } z \in D_{\rho} \\
\left|\langle v, \mathfrak{F} z\rangle_{W_{0}^{1,2}(\Omega, \rho d x)}\right| \leqslant C\|z\|_{H_{0}^{1}(\Omega)}
\end{array}\right\} .
$$

Проте, у загальному випадку, оператор $\mathfrak{F}^{*}$ не $€$ щільно визначений. Наступне твердження встановлює важливу властивість оператора $A$.

Теорема 4.1. Нехай $\rho: \Omega \rightarrow \mathbb{R}$ e ваговою функцією потенціального типу. Toдi

$$
\langle A(\mathfrak{F} z), \mathfrak{F} v\rangle_{\left(W_{0}^{1,2}(\Omega, \rho d x)\right)^{*} ; W_{0}^{1,2}(\Omega, \rho d x)}=\langle B(z), v\rangle_{H^{-1}(\Omega) ; H_{0}^{1}(\Omega)},
$$

$\partial e$

$$
\begin{gathered}
B(z)=-\triangle z-\frac{1}{2} V(x) z, \\
V(x)=-\triangle \ln \rho(x)-\frac{1}{2}|\nabla \ln \rho|_{\mathbb{R}^{N}}^{2},
\end{gathered}
$$

$i$ лінійний оператор $B$ визначае ізоморфізм простору $H_{0}^{1}(\Omega)$ в його дуальний nростір $H^{-1}(\Omega)$.

Доведення. Нехай $v$ та $z$-довільні елементи з $D_{\rho} \subset H_{0}^{1}(\Omega)$. Тоді, за наслідком 4.1, маємо $\mathfrak{F} z, \mathfrak{F} v \in W_{0}^{1,2}(\Omega, \rho d x)$. Далі, згідно з означенням оператора $\mathfrak{F}$, можна отримати таку низку перетворень:

$$
\begin{aligned}
A(\mathfrak{F} z) & =-\operatorname{div}\left(\rho A_{\theta} \nabla(\mathfrak{F} z)\right)=-\operatorname{div}\left(\rho \nabla\left(\frac{z}{\sqrt{\rho}}\right)\right) \\
& =-\rho^{1 / 2} \triangle z+\frac{1}{2} \rho^{-3 / 2}\left(\rho \triangle \rho-\frac{1}{2}|\nabla \rho|_{\mathbb{R}^{N}}^{2}\right) z \\
& =-\sqrt{\rho} \triangle z-\frac{1}{2} \sqrt{\rho} z\left(-\frac{\triangle \rho}{\rho}+\frac{1}{2} \frac{|\nabla \rho|_{\mathbb{R}^{N}}^{2}}{\rho^{2}}\right) \\
& =\sqrt{\rho}\left(-\triangle z-\frac{1}{2} z V(x)\right) .
\end{aligned}
$$

Оскільки

$$
\begin{aligned}
\frac{|\nabla \rho|_{\mathbb{R}^{N}}^{2}}{\rho^{2}} & =|\nabla \ln \rho|_{\mathbb{R}^{N}}^{2}, \\
\triangle \ln \rho & =\operatorname{div}(\nabla \ln \rho)=\operatorname{div}\left(\frac{\nabla \rho}{\rho}\right)=\frac{\triangle \rho \cdot \rho-(\nabla \rho, \nabla \rho)_{\mathbb{R}^{N}}}{\rho^{2}} \\
& =\frac{\triangle \rho \cdot \rho}{\rho^{2}}-\frac{|\nabla \rho|_{\mathbb{R}^{N}}^{2}}{\rho^{2}}=\frac{\triangle \rho}{\rho}-\frac{|\nabla \rho|_{\mathbb{R}^{N}}^{2}}{\rho^{2}},
\end{aligned}
$$


то функцію $V(x)$ можна подати у вигляді

$$
V(x)=-\triangle \ln \rho-\frac{1}{2} \frac{|\nabla \rho|_{\mathbb{R}^{N}}^{2}}{\rho^{2}}=-\triangle \ln \rho-\frac{1}{2}|\nabla \ln \rho|_{\mathbb{R}^{N}}^{2} .
$$

Таким чином,

$$
\begin{aligned}
\langle A(\mathfrak{F} z), \mathfrak{F} v\rangle_{\left(W_{0}^{1,2}(\Omega, \rho d x)\right)^{*} ; W_{0}^{1,2}(\Omega, \rho d x)} & \\
= & \left\langle-\operatorname{div}\left(\rho \nabla\left(\frac{z}{\sqrt{\rho}}\right)\right), \frac{v}{\sqrt{\rho}}\right\rangle_{\left(W_{0}^{1,2}(\Omega, \rho d x)\right)^{*} ; W_{0}^{1,2}(\Omega, \rho d x)} \\
= & \left\langle\rho^{1 / 2}\left(-\operatorname{div}(\nabla z)-\frac{1}{2} V(x) z\right), \frac{v}{\sqrt{\rho}}\right\rangle_{\left(W_{0}^{1,2}(\Omega, \rho d x)\right)^{*} ; W_{0}^{1,2}(\Omega, \rho d x)} \\
= & \left\langle-\triangle z-\frac{1}{2} V(x) z, v\right\rangle_{H^{-1}(\Omega) ; H_{0}^{1}(\Omega)}=\langle B(z), v\rangle_{H^{-1}(\Omega) ; H_{0}^{1}(\Omega)} .
\end{aligned}
$$

Насамкінець, оскільки $\rho \in$ функцією потенціального типу, то з (2.3) та нерівності Харді - Пуанкаре (див. [2,3])

$$
\int_{\Omega}\left[|\nabla y|_{\mathbb{R}^{N}}^{2}-\frac{\lambda_{*}}{K}\left(\sum_{i=1}^{K} \frac{1}{\left|x-x_{i}^{*}\right|_{\mathbb{R}^{N}}^{2}}\right) y^{2}\right] d x \geqslant C(\Omega) \int_{\Omega} y^{2} d x,
$$

випливає, що норми $\left(\int_{\Omega}\left[|\nabla y|_{\mathbb{R}^{N}}^{2}-\frac{1}{2} V(x) y^{2}\right] d x\right)^{1 / 2}$ та $\left(\int_{\Omega}|\nabla y|_{\mathbb{R}^{N}}^{2} d x\right)^{1 / 2}$ є еквівалентні в просторі $H_{0}^{1}(\Omega)$, а це означає, що оператор $B$ визначає ізоморфізм між $H_{0}^{1}(\Omega)$ та $H^{-1}(\Omega)$.

Беручи до уваги даний результат, перейдемо у початково-крайовій задачі (3.2)-(3.4) до її еквівалентного опису. Маємо таке твердження:

Твердження 4.2. $y \in L^{2}\left(0, T ; W_{0}^{1,2}(\Omega, \rho d x)\right)$ є розв'язком задачі $(3.2)-(3.4)$ тоді і тільки тоді, коли $y(t)=\frac{z(t)}{\sqrt{\rho}}$, де $z \in L^{2}\left(0, T ; H_{0}^{1}(\Omega)\right)$ задовольняе співвідношення

$$
\begin{aligned}
& \int_{\Omega} \dot{z}(t) w d x+\nu \int_{\Omega}(\nabla z(t), \nabla w)_{\mathbb{R}^{N}} d x-\nu \int_{\Omega} \frac{1}{2} V(x) z(t) w d x \\
& =\sum_{j=1}^{M} u_{j}(t) \int_{\Omega} \widehat{\mathcal{M}}_{j}(z(t)) w d x+\int_{\Omega} \frac{f(t)}{\sqrt{\rho}} w d x, \quad \forall w \in H_{0}^{1}(\Omega), \text { м.c. }(0, T) \\
& \lim _{t \rightarrow 0+}(z(t), \varphi)_{L^{2}(\Omega)}=\left(y_{0}, \varphi\right)_{L^{2}(\Omega)}, \quad \forall \varphi \in C_{0}^{\infty}(\Omega) .
\end{aligned}
$$

Тут $\widehat{\mathcal{M}}_{j}: L^{2}\left(0, T ; H_{0}^{1}(\Omega)\right) \rightarrow L^{2}\left(0, T ; H^{-1}(\Omega)\right)$ є лінійними операторами такими, що $\widehat{\mathcal{M}}_{j}(z)=\frac{1}{\sqrt{\rho}} \mathcal{M}_{j}(\mathfrak{F} z)$ для всіх $j=1, \ldots, M$, і при цьому існує стала величина $C>0$, яка забезпечує виконання наступних оцінок за всіх $z \in H_{0}^{1}(\Omega)$ :

$$
\left|\left\langle\widehat{\mathcal{M}}_{j}(z), z\right\rangle_{H^{-1}(\Omega) ; H_{0}^{1}(\Omega)}\right| \leqslant C\|z\|_{H_{0}^{1}(\Omega)}\|z\|_{L^{2}(\Omega)}, \quad \forall j=\overline{1, M} .
$$


Доведення. Беручи до уваги теорему 4.1 та залучаючи необхідіні міркування $3[2,3]$, достатньо зауважити, що для довільного $v \in W_{0}^{1,2}(\Omega, \rho d x)$ існує елемент $w \in H_{0}^{1}(\Omega)$ такий, що $v=\mathfrak{F} w:=\frac{w}{\sqrt{\rho}}$. Отже, мають місце наступні перетворення:

$$
\begin{aligned}
\int_{\Omega} f v \rho d x & =\int_{\Omega} f \frac{w}{\sqrt{\rho}} d x=\int_{\Omega} \frac{f}{\sqrt{\rho}} w d x, \quad \forall w \in H_{0}^{1}(\Omega), \\
\int_{\Omega}(\nabla y, \nabla v)_{\mathbb{R}^{N}} \rho d x & =\langle\operatorname{div}(\rho(x) \nabla y), v\rangle_{\left(W_{0}^{1,2}(\Omega, \rho d x)\right)^{*} ; W_{0}^{1,2}(\Omega, \rho d x)} \\
& =\langle\operatorname{div}(\rho(x) \nabla(\mathfrak{F} z)), \mathfrak{F} w\rangle_{\left(W_{0}^{1,2}(\Omega, \rho d x)\right)^{*} ; W_{0}^{1,2}(\Omega, \rho d x)} \\
& =\left\langle\operatorname{div}\left(\rho(x) \nabla\left(\frac{z}{\sqrt{\rho}}\right)\right), \frac{w}{\sqrt{\rho}}\right\rangle_{\left(W_{0}^{1,2}(\Omega, \rho d x)\right)^{*} ; W_{0}^{1,2}(\Omega, \rho d x)} \\
& =\left\langle\sqrt{\rho}\left(-\triangle z-\frac{1}{2} z V(x)\right), \frac{w}{\sqrt{\rho}}\right\rangle_{\left(W_{0}^{1,2}(\Omega, \rho d x)\right)^{*} ; W_{0}^{1,2}(\Omega, \rho d x)} \\
& =\left\langle-\triangle z-\frac{1}{2} V(x) z, w\right\rangle_{H^{-1}(\Omega) ; H_{0}^{1}(\Omega)} \\
& =\int_{\Omega}(\nabla z, \nabla w)_{\mathbb{R}^{N}} d x-\int_{\Omega} \frac{1}{2} V(x) z w d x, \\
\int_{\Omega} \mathcal{M}_{j}(v) w d x & \left.\left.=\int_{\Omega} \mathcal{M}_{j}(\mathfrak{F} z)\right) \mathfrak{F} w d x=\int_{\Omega} \frac{1}{\sqrt{\rho}} \mathcal{M}_{j}(\mathfrak{F} z)\right) w d x, \forall j=1, \ldots, M .
\end{aligned}
$$

Що торкається оцінок (4.8), то вони є прямим наслідком (3.6), твердження 4.1 та означення вагових просторів $W_{0}^{1,2}(\Omega, \rho d x)$ і $L^{2}(\Omega, \rho d x)$.

Беручи до уваги отримані результати, введемо до розгляду наступну задачу оптимального керування:

$$
J(u, z)=\int_{0}^{T}\left\|z-y_{a d}\right\|_{L^{2}(\Omega)}^{2} d t+\sum_{j=1}^{M} N_{j} \int_{0}^{T}\left|u_{j}(t)\right|^{2} d t \longrightarrow \inf
$$

за обмежень

$$
\begin{gathered}
\dot{z}-\nu \Delta z-\frac{\nu}{2} V(x) z=f \rho^{-\frac{1}{2}}(x)+\sum_{j=1}^{M} u_{j}(t) \widehat{\mathcal{M}}_{j}(z) \quad \text { в } Q=(0, T) \times \Omega, \\
z(t, x)=0 \text { на }(0, T) \times \partial \Omega \\
z(0, x)=y_{0} \quad \text { майже скрізь на } \Omega \\
u=\left[u_{1}, \ldots, u_{M}\right]^{T} \in U_{\partial} .
\end{gathered}
$$

Як буде показано далі, задачі оптимального керування (3.1)-(3.4) та (4.9)(4.13) є в певному сенсі еквівалентні. Дійсно, має місце такий результат.

Теорема 4.2. Нехай $\rho: \Omega \rightarrow \mathbb{R}_{+} \epsilon$ ваговою функиією потениіального тиny. Нехай $y_{a d} \in L^{2}(Q), f \in L^{2}\left(0, T ; L^{2}\left(\Omega, \rho^{-1} d x\right)\right)$ ma $y_{0} \in L^{2}(\Omega)$ е заданими 
функцілми, і нехай лінійні оператори $\mathcal{M}_{j}: W_{0}^{1,2}(\Omega, \rho d x) \rightarrow\left(W_{0}^{1,2}(\Omega, \rho d x)\right)^{*}$ підпорядковуються оцінкам (3.6). Тоді допустима пара $\left(u^{0}, z^{0}\right)$ е оптимальна в задачі (4.9)-(4.13) у тому і тільки у тому разі, коли

$$
\left(u^{0}, y^{0}\right)=\left(u^{0}, \mathfrak{F} z^{0}\right):=\left(u^{0}, \frac{z^{0}}{\sqrt{\rho}}\right)
$$

е розв'язком вихідної задачі оптимального керування (3.1)-(3.4). При цъому мае місце рівність

$$
\inf J(u, z)=J\left(u^{0}, z^{0}\right)=I\left(u^{0}, y^{0}\right)=\inf I(u, y) .
$$

Доведення. Справедливість даного результату легко встановити у спосіб, повністю ідентичний доведенню теореми 3.3 з [2]. При цьому потенціальність вагової функції $\rho$, а отже, справедливість неріності типу Харді - Пуанкаpe, та оцінки (4.8) гарантують (деталі див. [6, с.52]), що для довільних $f \in$ $L^{2}\left(0, T ; L^{2}\left(\Omega, \rho^{-1} d x\right)\right), y_{0} \in L^{2}(\Omega)$ та $u \in U_{\partial}$ існує єдиний розв'язок $z$ початковокрайової задачі (4.10)-(4.12) такий, що

$$
z \in W(0, T):=\left\{w: w \in L^{2}\left(0, T ; H_{0}^{1}(\Omega), \dot{w} \in L^{2}\left(0, T ; H^{-1}(\Omega)\right)\right\} .\right.
$$

\section{5. Теорема існування оптимального керування з апріорі заданою структурою оберненого зв'язку}

Доведемо розв'язність вихідної задачі оптимального керування (3.1)-(3.4).

Теорема 5.1. Нехай $\rho: \Omega \rightarrow \mathbb{R}_{+}$е ваговою функиією потенціального тиny. Hexaŭ $y_{a d} \in L^{2}(Q), f \in L^{2}\left(0, T ; L^{2}\left(\Omega, \rho^{-1} d x\right)\right)$, ma $y_{0} \in L^{2}(\Omega) \varepsilon$ заданими функиілми, i нехай лінійні неперервні оператори $\mathcal{M}_{j}: W_{0}^{1,2}(\Omega, \rho d x) \rightarrow$ $\left(W_{0}^{1,2}(\Omega, \rho d x)\right)^{*}$ підпорядковуються очінкам (3.6) та їх образи

$$
\widehat{\mathcal{M}}_{j}: L^{2}\left(0, T ; H_{0}^{1}(\Omega)\right) \rightarrow L^{2}\left(0, T ; H^{-1}(\Omega)\right)
$$

е компактні з простору $W(0, T)$ в $L^{2}\left(0, T ; H^{-1}(\Omega)\right)$. Тоді задача оптимального керування (3.1)-(3.4) має єдиний розв'язок

$$
\left(u^{0}, y^{0}\right) \in L^{2}(\Omega)^{M} \times L^{2}\left(0, T ; W_{0}^{1,2}(\Omega, \rho d x)\right) .
$$

Доведення. За теоремою 4.2 задачі (4.9)-(4.13) та (3.1)-(3.4) е еквівалентні. Отже, для однозначної розв'язності задачі (3.1)-(3.4) достатньо показати, що задача (4.9)-(4.13) має єдиний розв'язок. 
Нехай $\left\{\left(u^{k}, z^{k}\right)\right\}_{k=1}^{\infty}-$ довільна мінімізаційна послідовність для задачі (4.9)-(4.13), тобто $z^{k}:=z\left(u^{k}\right)$ є відповідними розв'язками задачі (4.10)-(4.12) і при цьому

$$
\lim _{k \rightarrow \infty}\left[\int_{0}^{T}\left\|z^{k}-y_{a d}\right\|_{L^{2}(\Omega)}^{2} d t+\sum_{j=1}^{M} N_{j} \int_{0}^{T}\left|u_{j}^{k}(t)\right|^{2} d t\right]=\inf J(u, z) \geqslant 0 .
$$

Отже, можемо вважати, що $\left\{u^{k}\right\}_{k \in \mathbb{N}} \in$ слабкозбіжна послідовність. Нехай $u^{*} \in L^{2}(0, T)^{M}-$ ï̈ слабка границя. Оскільки $\left\{u^{k}\right\}_{k \in \mathbb{N}} \subset U_{\partial}$, то, зважаючи на зроблені припущення щодо множини $U_{\partial}$ та за теоремою Мазура, отримуємо: $u^{*} \in U_{\partial}$. Покажемо, що відповідна послідовність розв'язків $\left\{z^{k}\right\}_{k \in \mathbb{N}} \in$ обмежена в просторі $W(0, T)$. Дійсно, для цього перепишемо співвідношення (4.10)-(4.12) у варіаційній формі

$$
\begin{aligned}
\left(\frac{d z^{k}(t)}{d t}\right. & , \phi)_{L^{2}(\Omega)}+\nu\left(\nabla z^{k}(t), \nabla \phi\right)_{L^{2}(\Omega)^{N}}-\frac{\nu}{2}\left(\phi, V z^{k}(t)\right)_{L^{2}(\Omega)} \\
& =\left(\frac{f}{\sqrt{\rho}}, \phi\right)_{L^{2}(\Omega)}+\sum_{j=1}^{M} u_{j}^{k}(t)\left(\widehat{\mathcal{M}}_{j}\left(z^{k}\right), \phi\right)_{L^{2}(\Omega)}, \quad \forall \phi \in H_{0}^{1}(\Omega) .
\end{aligned}
$$

Залучаючи нерівність (4.5) та властивість потенціальності функції ваги $\rho$, отримаємо:

$$
\begin{aligned}
\int_{\Omega}\left[|\nabla y|_{\mathbb{R}^{N}}^{2}\right. & \left.-\frac{1}{2} V(x) y^{2}\right] d x \stackrel{(2.3)}{\geqslant} \int_{\Omega}\left[|\nabla y|_{\mathbb{R}^{N}}^{2}-\frac{\lambda}{K}\left(\sum_{i=1}^{K} \frac{1}{\left|x-x_{i}^{*}\right|_{\mathbb{R}^{N}}^{2}}\right) y^{2}\right] d x \\
& =\left(1-\frac{\lambda}{\lambda_{*}}\right) \int_{\Omega}|\nabla y|_{\mathbb{R}^{N}}^{2} d x+ \\
& +\frac{\lambda}{\lambda_{*}} \int_{\Omega}\left[|\nabla y|_{\mathbb{R}^{N}}^{2}-\frac{\lambda_{*}}{K}\left(\sum_{i=1}^{K} \frac{1}{\left|x-x_{i}^{*}\right|_{\mathbb{R}^{N}}^{2}}\right) y^{2}\right] d x \\
& \stackrel{(4.5)}{\geqslant}\left(1-\frac{\lambda}{\lambda_{*}}\right) \int_{\Omega}|\nabla y|_{\mathbb{R}^{N}}^{2} d x+\frac{\lambda C(\Omega)}{\lambda_{*}} \int_{\Omega} y^{2} d x
\end{aligned}
$$

Покладемо тепер в $(5.2) \phi=z^{k}(t)$ і скористаємося попередньою нерівністю. Маємо:

$$
\begin{aligned}
& \frac{1}{2} \frac{d}{d t}\left\|z^{k}(t)\right\|_{L^{2}(\Omega)}^{2}+C_{1}\left\|z^{k}(t)\right\|_{H_{0}^{1}(\Omega)}^{2}+C_{2}\left\|z^{k}(t)\right\|_{L^{2}(\Omega)}^{2} \\
& \quad \leqslant C \sum_{j=1}^{M}\left|u_{j}^{k}(t)\right|\left\|z^{k}(t)\right\|_{L^{2}(\Omega)}\left\|z^{k}(t)\right\|_{H_{0}^{1}(\Omega)}+\left\|f(t) \rho^{-1 / 2}\right\|_{H^{-1}(\Omega)}\left\|z^{k}(t)\right\|_{H_{0}^{1}(\Omega)} \\
& \quad \leqslant \frac{C_{1}}{2}\left\|z^{k}(t)\right\|_{H_{0}^{1}(\Omega)}^{2}+C_{3} \sum_{j=1}^{M}\left|u_{j}^{k}(t)\right|^{2}\left\|z^{k}(t)\right\|_{L^{2}(\Omega)}^{2}+C_{4}\left\|f(t) \rho^{-1 / 2}\right\|_{H^{-1}(\Omega)}^{2}
\end{aligned}
$$


Оскільки $a b \leqslant \frac{\varepsilon}{2} a^{2}+\frac{1}{2 \varepsilon} b^{2}$ для довільних $\varepsilon>0$, то

$$
\begin{aligned}
\left\|z^{k}(t)\right\|_{L^{2}(\Omega)}^{2} & +C_{5} \int_{0}^{t}\left\|z^{k}(s)\right\|_{H_{0}^{1}(\Omega)}^{2} d s \\
& \leqslant C_{6} \int_{0}^{t}\left(1+\left|u^{k}(s)\right|_{\mathbb{R}^{M}}^{2}\right)\left\|z^{k}(s)\right\|_{L^{2}(\Omega)}^{2} d s \\
& +C_{6} \int_{0}^{t}\left\|f(s) \rho^{-1 / 2}\right\|_{H^{-1}(\Omega)}^{2} d s+\left|y_{0}\right|^{2} .
\end{aligned}
$$

В результаті, залучаючи інтегральну нерівність Гронуолла - Беллмана, приходимо до таких апріорних оцінок:

$$
\begin{aligned}
\left\|z^{k}(t)\right\|_{L^{2}(\Omega)}^{2} & \leqslant \widehat{C}:=\left[\left|y_{0}\right|^{2}+C_{6} \int_{0}^{T}\left\|f(s) \rho^{-1 / 2}\right\|_{H^{-1}(\Omega)}^{2} d s\right] \\
& \times \exp \left(C_{6}\left[\int_{0}^{T}\left(1+\left|u^{k}(s)\right|_{\mathbb{R}^{M}}^{2}\right) d s\right]\right), \\
\int_{0}^{T}\left\|z^{k}(s)\right\|_{H_{0}^{1}(\Omega)}^{2} d s & \leqslant \widehat{C} C_{6}^{-1}, \quad \forall k \in \mathbb{N} .
\end{aligned}
$$

Таким чином, послідовність $\left\{z^{k}\right\}_{k \in \mathbb{N}}$ є обмежена, а отже, і слабкокомпактна в просторі $W(0, T)$. Тому, з точністю до підпослідовності, можемо вважати, що існує елемент $z^{*} \in W(0, T)$ такий, що $z^{k} \rightarrow z^{*}$ слабко в $W(0, T)$. Беручи до уваги властивість компактності операторів $\widehat{\mathcal{M}}_{j}: H_{0}^{1}(\Omega) \rightarrow H^{-1}(\Omega)$, отримуємо:

$$
\widehat{\mathcal{M}}_{j}\left(z^{k}\right) \rightarrow \widehat{\mathcal{M}}_{j}\left(z^{*}\right) \quad \text { сильно в } L^{2}\left(0, T ; H^{-1}(\Omega)\right), \quad \forall j=\overline{1, M} .
$$

Об'єднуючи цей факт з властивістю слабкої збіжності керувань $u^{k} \rightarrow u^{*}$ в просторі $L^{2}(0, T)^{M}$, доходимо висновку:

$$
u_{j}^{k}(t) \widehat{\mathcal{M}}_{j}\left(z^{k}\right) \rightarrow u_{j}^{*}(t) \widehat{\mathcal{M}}_{j}\left(z^{*}\right) \quad \text { слабко в } \quad L^{1}\left(0, T ; H^{-1}(\Omega)\right), \quad \forall j=\overline{1, M} .
$$

Переходячи тепер до границі в інтегральній тотожності (5.2) з урахуванням властивостей (5.5)-(5.6), легко бачити, що пара $\left(u^{*}, z^{*}\right) \in$ допустима для задачі (4.9)-(4.13). Що стосується оптимальності цієї пари та її єдиності, то дана обставина є прямим наслідком співвідношення (5.1), строгої опуклості функціонала $J$ та його напівнеперервності знизу відносно збіжностей (5.5)-(5.6). Таким чином, за теоремою 4.2 , пара $\left(u^{*}, y^{*}\right):=\left(u^{*}, \frac{z^{*}}{\sqrt{\rho}}\right)$ є єдиний оптимальний розв'язок для задачі (3.1)-(3.4), що і потрібно було встановити.

Беручи до уваги умови теореми 5.1, доречно навести приклади операторів $\mathcal{M}_{j}: W_{0}^{1,2}(\Omega, \rho d x) \rightarrow\left(W_{0}^{1,2}(\Omega, \rho d x)\right)^{*}$, які таким умовам підпорядковуються.

Нехай $\left\{\Omega_{j}\right\}_{j=1}^{M}$ є заданою сукупністю відкритих підмножин множини $\Omega$ з ненульовою лебеговою мірою. Покладемо

$$
\mathcal{M}_{j}(\phi)=\frac{\sqrt{\rho(x)}}{\left|\Omega_{j}\right|} \int_{\Omega_{j}} \phi(s) \sqrt{\rho}(s) d s, \quad \forall j=1, \ldots, M .
$$


Покажемо для початку, що $\mathcal{M}_{j}: W_{0}^{1,2}(\Omega, \rho d x) \rightarrow\left(W_{0}^{1,2}(\Omega, \rho d x)\right)^{*}$. Дійсно, оскільки дуальний простір $\left(W_{0}^{1,2}(\Omega, \rho d x)\right)^{*}$ можна утотожнити з простором $W^{-1,2}\left(\Omega, \rho^{-1} d x\right)$, то типовим представником простору $\left(W_{0}^{1,2}(\Omega, \rho d x)\right)^{*} \in$ такий функціонал:

$$
\begin{aligned}
& <F, y>_{\left(W_{0}^{1,2}(\Omega, \rho d x)\right)^{*}, W_{0}^{1,2}(\Omega, \rho d x)}=\int_{\Omega} f_{0} y d x+\sum_{i=1}^{N} \int_{\Omega} f_{i} D_{i} y d x \leqslant \\
& \leqslant\left(\int_{\Omega} f_{0}^{2} \rho^{-1} d x\right)^{1 / 2}\|y\|_{L^{2}(\Omega, \rho d x)}+\sum_{i=1}^{N}\left(\int_{\Omega} f_{i}^{2} \rho^{-1} d x\right)^{1 / 2}\left\|D_{i} y\right\|_{L^{2}(\Omega, \rho d x)} \leqslant \\
& \leqslant \sum_{i=0}^{N}\left(\int_{\Omega} f_{i}^{2} \rho^{-1} d x\right)^{1 / 2}\|y\|_{W_{0}^{1,2}(\Omega, \rho d x)},
\end{aligned}
$$

Отже, виходячи з (5.7), маємо:

$$
\begin{aligned}
\left\|\mathcal{M}_{j}(\phi)\right\|_{\left(W_{0}^{1,2}(\Omega, \rho d x)\right)^{*}}^{2} & \leqslant \int_{\Omega}\left(\frac{\sqrt{\rho(x)}}{\left|\Omega_{j}\right|} \int_{\Omega_{j}} \phi(s) \sqrt{\rho}(s) d s\right)^{2} \frac{1}{\rho} d x \\
& \leqslant \frac{1}{\left|\Omega_{j}\right|^{2}} \int_{\Omega} \int_{\Omega_{j}} \phi^{2}(s) \rho(s) d s \int_{\Omega_{j}} d s d x \\
& \leqslant \frac{|\Omega|}{\left|\Omega_{j}\right|}\|\phi\|_{L^{2}(\Omega, \rho d x)}^{2} \leqslant \frac{|\Omega|}{\left|\Omega_{j}\right|}\|\phi\|_{W_{0}^{1,2}(\Omega, \rho d x)}^{2},
\end{aligned}
$$

що означає $\mathcal{M}_{j} \in \mathcal{L}\left(W_{0}^{1,2}(\Omega, \rho d x),\left(W_{0}^{1,2}(\Omega, \rho d x)\right)^{*}\right)$ за всіх $j=1, \ldots, M$.

Тепер покажемо, що оператори $\widehat{\mathcal{M}}_{j}: L^{2}\left(0, T ; H_{0}^{1}(\Omega)\right) \rightarrow L^{2}\left(0, T ; H^{-1}(\Omega)\right) \in$ компактні з простору $W(0, T)$ в $L^{2}\left(0, T ; H^{-1}(\Omega)\right)$. Дійсно, за побудовою маємо (див. твердження 4.2)

$$
\widehat{\mathcal{M}}_{j}(z)=\frac{1}{\sqrt{\rho}} \mathcal{M}_{j}(\mathfrak{F} z)=\frac{1}{\left|\Omega_{j}\right|} \int_{\Omega_{j}} z(s) d s, \quad \forall j=1, \ldots, M, \forall z \in H_{0}^{1}(\Omega) .
$$

Отже, якщо послідовність $\left\{z^{k}\right\}_{k \in \mathbb{N}}$ Збігається слабко в $W(0, T)$ до деякого елемента $z^{*} \in W(0, T)$, то за теоремою про компактне вкладення простору $W(0, T)$ в $L^{2}\left(0, T ; L^{2}(\Omega)\right)$ (див. [4]) отримуємо:

$$
z^{k} \rightarrow z^{*} \text { сильно в } L^{2}\left(0, T ; L^{2}(\Omega)\right) .
$$

Таким чином, має місце наступна збіжність числової послідовності:

$$
\widehat{\mathcal{M}}_{j}\left(z^{k}\right):=\frac{1}{\left|\Omega_{j}\right|} \int_{\Omega_{j}} z^{k}(s) d s \longrightarrow \frac{1}{\left|\Omega_{j}\right|} \int_{\Omega_{j}} z^{*}(s) d s=\widehat{\mathcal{M}}_{j}\left(z^{*}\right),
$$

що означає:

$$
\widehat{\mathcal{M}}_{j}\left(z^{k}\right) \rightarrow \widehat{\mathcal{M}}_{j}\left(z^{*}\right) \text { сильно в } L^{2}\left(0, T ; H^{-1}(\Omega)\right) .
$$


Отже, властивість компактності встановлено.

\section{6. Геометричне узагальнення задачі оптимального керування з апріорі заданою структурою оберненого зв'язку}

Нехай всюди в цьому параграфі $E$ є замкнена непорожня вимірна підмножина множини $\Omega$ така, що dist $(E, \partial \Omega) \geqslant r>0.3$ довільною точкою $b \in E$ далі будемо пов'язувати відкриту кулю $B(b, r)=\left\{x \in \mathbb{R}^{N}:|x-b|<r\right\}$. Нехай $c^{-1}=|B(b, r)| \in$ лебегова міра такої кулі. Введемо до розгляду таку сукупність лінійних операторів:

$$
\mathcal{M}_{j}(\phi)=c \sqrt{\rho(x)} \int_{B\left(b_{j}, r\right)} \phi(s) \sqrt{\rho}(s) d s, \quad \forall j=1, \ldots, M, \quad \forall \phi \in W_{0}^{1,2}(\Omega, \rho d x),
$$

де $\left\{b_{1}, \ldots, b_{M}\right\}$ є деяка система точок в $E$. Як легко бачити (див. попередній параграф),

$$
\mathcal{M}_{j} \in \mathcal{L}\left(W_{0}^{1,2}(\Omega, \rho d x),\left(W_{0}^{1,2}(\Omega, \rho d x)\right)^{*}\right), \quad \forall j=1, \ldots, M
$$

Розглянемо тепер узагальнення задачі оптимального керування (3.1)-(3.4), суттєва відмінність якого від постановки (3.1)-(3.4) полягає в тому, що "позиціювання" $b_{j} \in E$ апріорно заданого закону оберненого звязку у формі (3.5) $є$ невідоме і підлягає визначенню:

$$
\begin{gathered}
I(u, b, y)=\int_{0}^{T}\left\|y(t, \cdot)-\frac{y_{a d}(t, \cdot)}{\sqrt{\rho}}\right\|_{L^{2}(\Omega, \rho d x)}^{2} d t+\sum_{j=1}^{M} N_{j} \int_{0}^{T}\left|u_{j}(t)\right|^{2} d t \rightarrow \inf \\
\rho(x) \dot{y}-\nu \operatorname{div}(\rho(x) \nabla y)=f(t, x)+p(t, x) \quad \text { в } Q=(0, T) \times \Omega \\
y(t, x)=0 \text { на }(0, T) \times \partial \Omega \\
\sqrt{\rho(x)} y(0, x)=y_{0} \quad \text { майже скрізь на } \Omega \\
p(t, x)=\sum_{j=1}^{M} u_{j}(t) \mathcal{M}_{j}(y)=c \sqrt{\rho(x)} \sum_{j=1}^{M} u_{j}(t) \int_{B\left(b_{j}, r\right)} y(t, s) \sqrt{\rho}(s) d s \\
u=\left[u_{1}, \ldots, u_{M}\right]^{T} \in U_{\partial}, \quad b=\left[b_{1}, \ldots, b_{M}\right]^{T} \in E^{M}
\end{gathered}
$$

Тут $N_{j}>0$ - задані сталі; $U_{\partial}-$ непорожня опукла замкнена підмножина в $L^{2}(0, T)^{M}$.

У повній аналогії до теореми 4.2 можна показати, що задача (6.2)-(6.7) є еквівалентна (в сенсі бієкції $(u, b, z) \mapsto(u, b, \mathfrak{F} z))$ наступній задачі оптималь- 
ного керування:

$$
\begin{gathered}
J(u, b, z)=\int_{0}^{T}\left\|z-y_{a d}\right\|_{L^{2}(\Omega)}^{2} d t+\sum_{j=1}^{M} N_{j} \int_{0}^{T}\left|u_{j}(t)\right|^{2} d t \longrightarrow \text { inf }, \\
\dot{z}-\nu \Delta z-\frac{\nu}{2} V(x) z=f \rho^{-\frac{1}{2}}(x)+q(t, x) \quad \text { в } Q=(0, T) \times \Omega, \\
z(t, x)=0 \text { на }(0, T) \times \partial \Omega, \\
z(0, x)=y_{0} \text { майже скрізь на } \Omega, \\
q(t, x)=c \sum_{j=1}^{M} u_{j}(t) \int_{B\left(b_{j}, r\right)} z(t, s) d s, \\
u=\left[u_{1}, \ldots, u_{M}\right]^{T} \in U_{\partial}, \quad b=\left[b_{1}, \ldots, b_{M}\right]^{T} \in E^{M} .
\end{gathered}
$$

Для доведення розв'язності задачі (6.8)-(6.13) скористаємося відомими результатами теорії оптимального керування системами з розподіленими параметрами (див., напр., [5,6]).

Теорема 6.1. Нехай $\rho: \Omega \rightarrow \mathbb{R}_{+} \varepsilon$ ваговою функиією потенціального тиny. Hexaü $y_{a d} \in L^{2}(Q), f \in L^{2}\left(0, T ; L^{2}\left(\Omega, \rho^{-1} d x\right)\right)$, ma $y_{0} \in L^{2}(\Omega)$ e заданими функиіями. Нехай $Е$ е замкненою непорожнъою вимірною підмножиною множини $\Omega$ такою, що dist $(E, \partial \Omega) \geqslant r>0$. Тоді задача оптимального керування (6.2)-(6.7) е розв'язна, тобто існуе принаймні один набір функuій $\left(u^{0}, b^{0}, y^{0}\right)$ у просторі $L^{2}(\Omega)^{M} \times E^{M} \times L^{2}\left(0, T ; W_{0}^{1,2}(\Omega, \rho d x)\right)$ такий, що $u^{0} \in U_{\partial}, b^{0} \in E^{M}, y^{0}=y\left(u^{0}, b^{0}\right)$ i при ивому

$$
J\left(u^{0}, b^{0}, y^{0}\right) \leqslant J(u, b, y), \quad \text { для всіх допустимих наборів }(u, b, y) .
$$

Доведення. Нехай $\left\{\left(u^{k}, b^{k}, z^{k}\right)\right\}_{k=1}^{\infty}-$ довільна мінімізаційна послідовність для задачі (6.8)-(6.13). Отже, $u^{k} \in U_{\partial}, b^{k} \in E^{M}, z^{k}=z\left(u^{k}, b^{k}\right) \in$ відповідними розв'язками задачі (6.9)-(6.11) і при цьому

$$
\lim _{k \rightarrow \infty}\left[\int_{0}^{T}\left\|z^{k}-y_{a d}\right\|_{L^{2}(\Omega)}^{2} d t+\sum_{j=1}^{M} N_{j} \int_{0}^{T}\left|u_{j}^{k}(t)\right|^{2} d t\right]=\inf J(u, b, z) \geqslant 0 .
$$

Оскільки множина $E \in$ обмежена та замкнена, то знайдеться вектор $b^{*}=$ $\left[b_{1}^{*}, \ldots, b_{M}^{*}\right] \in E^{M}$ такий, що з точністю до підпослідовності маємо:

$$
b^{k} \rightarrow b^{*} \quad \text { в } \quad \mathbb{R}^{M N} \text { за } k \rightarrow \infty .
$$

Зважаючи на те, що величина $c$ в (6.12) $\left(c^{-1} \in\right.$ лебеговою мірою множин $\left.B\left(b_{j}^{k}, r\right) \cap \Omega\right)$ не залежить від вибору центрів $b_{j}^{k} \in E$, можемо скористатися аргументами з доведення теореми 5.1 та отриманими там апріорними оцінками щодо компактності послідовностей $\left\{u^{k}\right\}_{k \in \mathbb{N}} \subset U_{\partial}$ та $\left\{z^{k}\right\}_{k \in \mathbb{N}} \subset W(0, T)$. 
Отже, існують елементи $u^{*} \in U_{\partial}$ та $z^{*} \in W(0, T)$ такі, що мають місце слабкі збіжності (на відповідних підпослідовностях)

$$
z^{k} \rightarrow z^{*} \quad \text { в } W(0, T), \quad u^{k} \rightarrow u^{*} \text { в } L^{2}(0, T)^{M} .
$$

Покажемо, що граничні елементи пов'язані співвідношенням $z^{*}=z\left(u^{*}, b^{*}\right)$. Це означає, що $z^{*} \in$ розв' язком початково-крайової задачі (6.9)-(6.11) за $u=$ $u^{*}$ та $b=b^{*}$. Дійсно, як випливає із структури інтегральної тотожності (5.2), для цього досить встановити, що за кожного значення індекса $j=1, \ldots, M$ має місце наступна збіжність:

$$
u_{j}^{k}(t) \int_{B\left(b_{j}^{k}, r\right)} z^{k}(t, s) d s \rightarrow u_{j}^{*}(t) \int_{B\left(b_{j}^{*}, r\right)} z^{*}(t, s) d s \quad \text { слабко в } L^{1}(Q) .
$$

Проте, беручи до уваги властивість $(6.16)_{2}$, для виконання умови (6.17) досить гарантувати, що

$$
\int_{B\left(b_{j}^{k}, r\right)} z^{k}(t, s) d s \rightarrow \int_{B\left(b_{j}^{*}, r\right)} z^{*}(t, s) d s \text { сильно в } L^{2}(Q) .
$$

3 цією метою зауважимо таке: оскільки

$$
\begin{aligned}
\int_{B\left(b_{j}^{k}, r\right)} z^{k}(t, s) d s & -\int_{B\left(b_{j}^{*}, r\right)} z^{*}(t, s) d s \\
& =\int_{B\left(b_{j}^{k}, r\right)}\left(z^{k}(t, s)-z^{*}(t, s)\right) d s \\
& -\int_{B\left(b_{j}^{*}, r\right) \Delta B\left(b_{j}^{k}, r\right)} z^{*}(t, s) d s=g^{k}+h^{k}
\end{aligned}
$$

де позначено

$$
B\left(b_{j}^{*}, r\right) \Delta B\left(b_{j}^{k}, r\right)=\left[B\left(b_{j}^{*}, r\right) \backslash B\left(b_{j}^{k}, r\right)\right] \cup\left[B\left(b_{j}^{k}, r\right) \backslash B\left(b_{j}^{*}, r\right)\right],
$$

то, беручи до уваги компактність вкладення $W(0, T) \hookrightarrow L^{2}\left(0, T ; H^{-1}(\Omega)\right)$ та властивість $(6.16)_{1}$, маємо:

$$
\begin{aligned}
\int_{Q}\left|g^{k}\right|^{2} d z d t=\int_{0}^{T} & \int_{\Omega}\left(\int_{B\left(b_{j}^{k}, r\right)}\left(z^{k}(t, s)-z^{*}(t, s)\right) d s\right)^{2} d x d t \\
& \leqslant|\Omega|^{2} \int_{0}^{T} \int_{\Omega}\left|z^{k}(t, x)-z^{*}(t, x)\right|^{2} d x d t \rightarrow 0 \quad \text { за } k \rightarrow \infty .
\end{aligned}
$$

Що стосується відхилення $h^{k}$, то, виходячи з геометричних міркувань, маємо таку оцінку для лебегової міри множин (6.19):

$$
\left|B\left(b_{j}^{*}, r\right) \Delta B\left(b_{j}^{k}, r\right)\right| \leqslant C^{*}\left|b_{j}^{k}-b_{j}^{*}\right|_{\mathbb{R}^{N}}
$$


де стала $C^{*}$ не залежить від положення центрів куль $b_{j}^{k}$ та $b_{j}^{*}$. Отже, за нерівністю Коші - Буняковського та властивістю (6.15), доходимо висновку:

$$
\begin{aligned}
\int_{Q}\left|h^{k}(t, x)\right|^{2} d x d t & =\int_{0}^{T} \int_{\Omega}\left(\int_{B\left(b_{j}^{*}, r\right) \Delta B\left(b_{j}^{k}, r\right)} z^{*}(t, s) d s\right)^{2} d x d t \\
& \leqslant C^{*}\left|b_{j}^{k}-b_{j}^{*}\right|_{\mathbb{R}^{N}} \int_{0}^{T} \int_{\Omega}\left(\int_{\Omega}\left|z^{*}(t, x)\right|^{2} d s\right) d x d t \\
& =C^{*}|\Omega|\left\|z^{*}\right\|_{L^{2}(Q)}^{2}\left|b_{j}^{k}-b_{j}^{*}\right|_{\mathbb{R}^{N} \rightarrow 0 \quad \text { за } \quad k \rightarrow \infty}
\end{aligned}
$$

Таким чином, умови (6.18), а отже, і (6.17), встановлено. Отриманий результат дозволяє перейти до границі в інтегральній тотожності (5.2) за $k \rightarrow \infty$ i показати, що трійка елементів $\left(u^{*}, b^{*}, z^{*}\right)$ є допустима для задачі (6.8)-(6.13). Що стосується оптимальності цього розв' язку, то дана обставина є прямим наслідком співвідношення (6.14) та властивості напівнеперервності знизу функціонала $J$ відносно збіжностей (6.15)-(6.16). Таким чином, за теоремою 4.2, керування $\left(u^{*}, b^{*}\right)$ є оптимальне і для задачі $(6.2)-(6.7)$, що і потрібно було встановити.

\section{Бібліографічні посилання}

1. Баланенко I. Г. Про класифікацію розв'язків початково-крайових задач для вироджених параболічних рівнянь/ І. Г. Баланенко, П. І. Когут // Вісник ДНУ. Серія: Моделювання.- Д. : Вид-во ДНУ. - 2011, Вип. 3, № 8.- С. 55-73.

2. Баланенко I. Г. Про одну задачу оптимального керування для виродженого параболічного рівняння/ I. Г. Баланенко, П. І. Когут // Вісник ДНУ. Серія: Моделювання.- Д. : Вид-во ДНУ. - 2012, Вип. 4, № 8.- С. 3-18.

3. Баланенко I. Г. Про одну задачу оптимального керування для виродженого параболічного рівняння/ І. Г. Баланенко, П. І. Когут // Вісник ДНУ. Серія: Моделювання.- Д. : Вид-во ДНУ. - 2013, Вип. 5, № 8.- С. 47-61.

4. Иваненко В. И. Вариационные методы в задачах управления для систем с распределенными параметрами/ В. И. Иваненко, В. С. Мельник.- К. : Наукова думка, 1988. - 324 с.

5. Lions J.-L. Optimal Control of Systems Governed by Partial Differential Equations/ J.-L. Lions.- Berlin, Springer-Verlag, 1971.

6. Lions J.-L. Some Aspects of the Optimal Control of Distributed Parameters Systems / J.-L. Lions.- Philadelpfia, Society for Industrial and Applies Mathematics, 1972.

7. Vazquez J. L. The Hardy inequality and the asymptotic behaviour of the heat equation with an inverse-square potential/ J. L.. Vazquez, E. Zuazua // J. of Functional Analysis. - 2000. - Vol. 173. - P. 103-153. 